\title{
A Review Paper on Solar Tracking System for Photovoltaic Power Plant
}

\author{
Bhagwan Deen Verma \\ School of Energy and Environment Management \\ Rajiv Gandhi Proudyogiki Vishwavidyalaya, \\ Bhopal, India
}

\author{
Prof. (Dr.) Mukesh Pandey \\ School of Energy and Environment Management \\ Rajiv Gandhi Proudyogiki Vishwavidyalaya, \\ Bhopal, India
}

\author{
Asst. Prof. Anurag Gour \\ School of Energy and Environment Management \\ Rajiv Gandhi Proudyogiki Vishwavidyalaya, \\ Bhopal, India
}

\begin{abstract}
In today's era the biggest problem World is facing is energy crises and we know that fossil fuels are available in very limited amount. Also there overuse in last 30-40 years has reduced them further. So, now to meet our energy demands the only option we are left is to utilize the Renewable resources of energy that is available in abundance. There are various sources of renewable energy like wind, sun and geothermal but most cost effective among them is Solar Energy. Solar energy can not only meet our existing energy demands but can also provide us clean and cheap energy. Solar Panels once installed can give energy for several years without having any maintenance cost. Solar photovoltaic systems are such systems which are used for harnessing solar energy but we since the earth is rotating around the sun due to which solar energy in existing Solar panels is available only for a limited time throughout the day. To overcome this problem solar trackers are used. Authors in this study have tried to explore the possibility of solar trackers and their cost effectiveness in solar photovoltaic.
\end{abstract}

Keywords- Photovoltaic, solar Tracker, Renewable Energy Sources (RES), STS (Solar Tracker System)

\section{INTRODUCTION}

The shift from conventional fossil-based resources several solar power resources are available in like geothermal energy, wind energy, bio-energy as well as solar power. Solar energy is among the most significant attempting to solve the issue of worldwide heating in addition to is a source of fresh energy. it may also assist in provided that an alternative so sensational fossil-based energy resources in addition to as a cost-effective source of energy besides. Consumption is actually tined enclosed by photovoltaic and thermal energy, making use of solar panels as well as solar collectors, respectively. there will be numerous applications given that photovoltaic thermal sporting goods. At low-temperature ranges, space conditioning and domestic hot water by-product tend to be the overall most typical use. at medium temperature ranges, solar temperature reduction, desalinisation furthermore postindustrial process high temperature tend to purpose which can deed alternative energy. at high-temperature stages, concentrating solar power plants are going to be sensational programs that catch the overall most attention of worldwide. furthermore, for very high-temperature ranges up to $1000{ }^{\circ} \mathrm{c}$, processes specified hydrogen production in addition to methanol-reforming can use sun irradiation [1].

Accompanied by all of the anticipated res, solar energy (SPV) systems experience has taken major toleration in addition to investment funds wondering its around the world potential in addition to its totipotent to set off-world power very little time. so, within the coming years, SPV techniques will experience the highest participation in energy production among all of the res. moreover, the SPV module is really a dc source, something that often sets off powerful dc whose amplitude is dependent on the availability containing daytime sunlight as well as the temperature at standard test condition (STC) consisting of $25^{\circ} \mathrm{c}$ along with Solar Constant of $1 \mathrm{~kW} / \mathrm{m}^{2}$ [2]. Significantly, the SPV module indicates the overall unidirectional characteristic. Hence, the power output of the SPV module is usually withstanding broadens along with atmospheric temperature along with room temperature. Also, the SPV generator indicates only one MPPT under full exposure to sunlight.

On this context, the main objective consisting of utilising the MPPT tracking method is to exactly track and distinguish the overall global MPPT, as well as thereby gathering the overall maximum power. Additionally, MPPT techniques are compared in order to as an organised set of rules that is normally needed in order to operate on the system with efficiency. This signified, multiple MPPT tracking algorithms have already been published in the scientific literature, which is, actually, the heterogeneous ways in order to match the source and load impedances [3]. The correct geographical orientation of such devices would possibly raise the light intensity of the incident solar radiation flux, and that depends upon azimuth and inclination angles.

\subsection{SOLAR ENERGY}

The overall energy that's harnessed using sun radiation is known as solar energy. Radiant light and heat from the powerful sun have a tendency to harnessed exploitation various kinds of technologies like solar heating, solar cell, solar thermal thermoelectric, solar architecture and artificial photosynthesis. It's estimated that earth gets approx 
$1 \mathrm{~kW} / \mathrm{m}^{2}$ at high noon but also direct changeover from photon energy to electricity already done and solar power plants are now setting up on considerable Scale. Nevertheless, solar energy production competence is not satisfactory. Therefore it really is preferable to extract the maximum amount of energy as feasible by means of lowering losses. Solar energy seems to have benefits over all other sources of energy. The most important fact is, except the overall a lot of installation cost and zero maintenance cost it truly is quite free of cost and with no energy restriction. it does not reject heat and carbon dioxide or radioactive particles, as well as solar energy, does no longer produce any noise. As a result, it's totally most environment-friendly. Solar energy serves as a reliable source of energy in the hilly parts of the world along with isolated lands where transmission via power line are often costly or very unlikely. Photovoltaic panels have a tendency to be used in the backcountry for electrification [4]. In addition, urbanised electrification with the aid of street lights by a photovoltaic panel is becoming popular. Modern electrical gadgets also have the facility to run along with solar power. These days' electric vehicles, the building-integrated solar system as well as smart grid system have begun implementing solar energy broadly. Spacecraft, as well as satellites that are sent to space for a long time, have no other alternative energy other than solar.

\subsection{Photovoltaic Technology}

Solar Cells are the best-known technique for producing electrical power through Silicon Cells swaddle in photovoltaic modules. to elucidate the photovoltaic solar panel more easily, photons from sunlight energies electrons in an upper state of energy and coming to the lower state to release one electron so that creating electricity. The term photovoltaic defines the general unbiased working condition of a photodiode in which current through the appliance is due to the transduced light energy [5]. Solar cells generate direct current electricity from light, which can be used to recharge a battery for electric devices.

\subsection{Solar Module}

In the field of photovoltaic, a photovoltaic module or photovoltaic panel is a packaged interconnected assembly of photovoltaic cells, also known as solar cells. An installation of photovoltaic modules or panels is known as a photovoltaic array. Photovoltaic cells typically require protection from the environment. For cost and practicality reasons a number of cells are connected electrically and packaged in a photovoltaic module, while a collection of these modules that are mechanically fastened together, wired, and designed to be a field-installable unit, sometimes with a glass covering and a frame and backing made of metal, plastic or fiberglass, are known as a photovoltaic panel or simply solar panel. The foremost application of photovoltaics was to power satellite as well as spacecraft, but nowadays the majority of solar modules are going to be used for grid-connected power generation [6]. Therefore, the inverter is needed to transform the dc to ac. there's a less significant marketplace for off-grid power for inaccessible outbuildings, wayside difficulty in communication, meteorological, as well as cathodic protection are needed to be in pipelines.

\subsection{Solar Tracker Fundamentals}

A solar tracker is a device for aligning a day lighting reflector, solar photovoltaic panel or concentrating solar reflector or lens toward the sun. The sun's position in the sky varies with the seasons (elevation) even with the time of day as the sun moves across the sky. Solar-powered devices function best when every time pointed at the sun, so a solar tracker will hike the effectively of devices over any confirmed position, at the cost of extra system complexity. There are several kinds of solar trackers, of different charges, quality, and performance. Some best-known type of solar tracker is the heliostat, a movable mirror which reflects the moving sun to a fixed position, but several other techniques will be used as well. The efficiency of the solar tracker depends upon the application. concentrators, in particular in solar cell processes, require an intensity of exactness to ensure that fact that saturated sunlight is directed exactly to the device, that is at (or near) the focal point of the reflector [3]. Usually, concentrator techniques won't work without tracking, so at slightest single-axis tracking is necessary. Sizeable power plants or high-temperature resources research facilities using multiple ground-mounted mirrors along with an absorbent spot need with high accuracy similar to that used for solar telescopes [7].

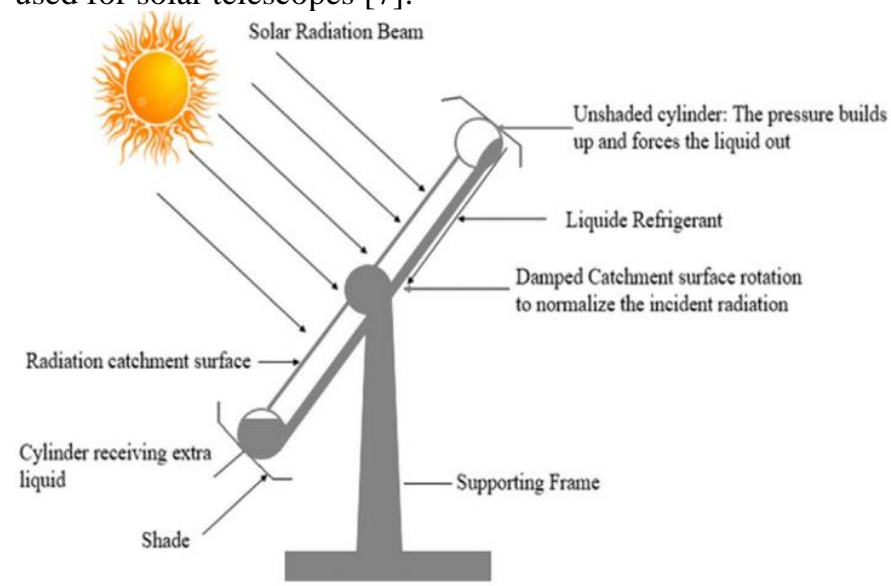

Figure 1: Basic design of solar tracker

\subsection{Sun Path, Azimuth \& Altitude Angle}

The change of position of moving sun varies at different time and season to season due to earth's continuous and periodic rotation and revolution. As a result it has become necessary to locate the orientation of sun for a particular moment. The locations are placed on a special type of chart named Sun Path Diagram. A Sun Path Diagram shows the azimuth angle, elevation angle, sun paths throughout the years, sunrise and sunset time etc. 


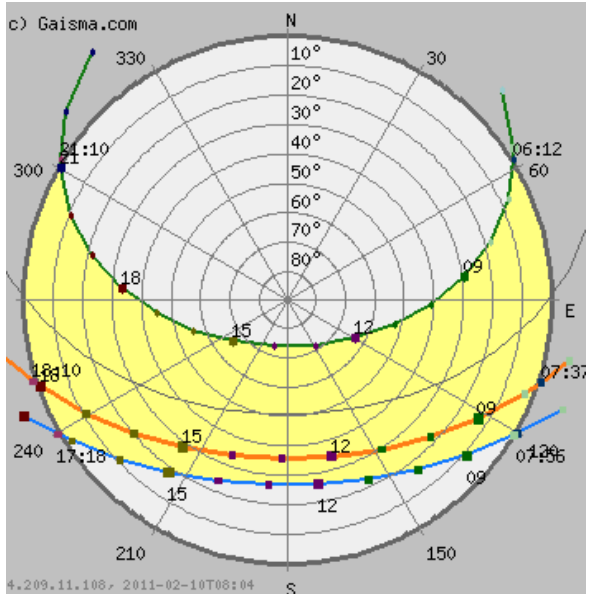

Figure 2: Sun path diagram

1.5.1 Solar azimuth, $\psi$, is the direction of the sun from the observer, expressed because of the hour angle from the north point of the line to the point at which a vertical circle passing through the sun intersects the horizon.

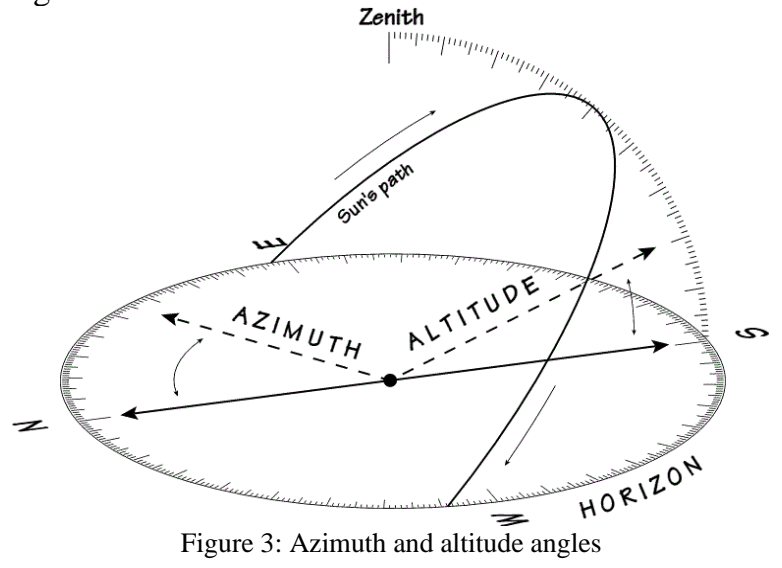

1.5.2 Solar altitude, $\alpha$, is the angular height of the sun measured from the horizon. Above the horizon is positive, below is negative. The sun directly in the centre of the sky has a Solar Altitude of 90 degrees.

1.5.3 The declination angle, $\delta$, is the angle of deviation of the sun from directly above the equator. This angle can be mathematically calculated by the following equation:

$$
\delta=23.45 \operatorname{Sin}(360(n-180) / 365)
$$

Here, $n$ is the number specified for the days within the year and $\delta$ is positive for the angles north to the equator and negative for the angles south to the equator.

1.5.4 The zenith angle, $v_{z}$, is the angle between the sun and a line perpendicular to the earth's surface. It is the complement of solar altitude. The zenith angle is given by the following equation:

$$
\mathrm{v}_{\mathrm{z}}=\Phi-\delta
$$

Here, $\phi$ is the latitude in degree.

\subsubsection{Solar Irradiance}

Solar irradiance is the measure of the power density of sunlight. Its unit is $\mathrm{W} / \mathrm{m}^{2}$ and is an instantaneous quantity. The irradiance received by the earth from solar radiation is $1367 \mathrm{~W} / \mathrm{m}^{2}$. After getting absorbed by atmosphere, as it passes through it, the radiation becomes $1000 \mathrm{~W} / \mathrm{m}^{2}$ at surface. Solar radiation is affects the solar cell output performance and so it is a very important factor in this field. Sunlight consist energy which has wavelengths with in a wide range of electromagnetic wave spectrum. But no solar cell can absorb energy from the whole spectrum [8]. A solar cell is mainly designed for absorption of a portion of the total radiation spectrum. Photovoltaic solar cells are designed for the absorption of the visible spectrum only. The direct normal solar irradiance is given by,

$$
I_{t, \mathrm{n}}=\mathbf{I}_{\mathrm{b}, \mathrm{n}} \cos _{\mathrm{z}}+\mathbf{I}_{\mathrm{d}, \mathrm{n}}
$$

Here,

$I_{t, n}=$ the irradiance coming directly from the sun $I_{d, n}=$ the diffuse radiation

Solar irradiance is a parameter directly related to solar energy received by solar panel and maximum when rays are perpendicular to panel plane. To achieve maximum solar energy it is necessary to rotate the panel face towards the sun. The device that is used for this purpose is called Solar Tracker.

\subsection{Various type of tracking}

Solar tracking system is classified by its degrees of rotation. According to degrees of rotation trackers can be grouped into two primary categories

\section{- Single Axis Solar Tracker \\ - Dual Axis Solar Tracker}

\subsubsection{Single Axis Solar Tracker}

A single-axis solar tracker continues to follow the movement of the sun either horizontally or vertically. As the name recommends, this sort of tracker seems to have just one axis for rotary motion. The horizontal variety of solar tracker is utilized in tropical regions where the sun will receive very way up at midday, except the days are going to be short. On the other hand, the upright kind of solar tracker is employed in regions with elevations where the sun doesn't have to get high, but summer days are often lengthy. In concentrated solar power processes, single-axis trackers are going to be used with flat surface solar module [9].

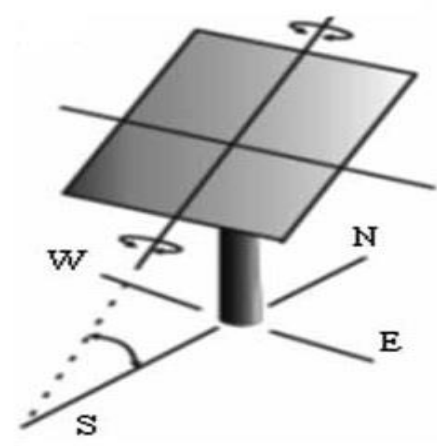

Figure 4: Single Axis Solar Tracker 


\subsubsection{Dual Axis Solar Tracker}

A dual-axis solar tracker seems to have two degrees of rotary motion. it could possibly track the sun either horizontally and vertically. This type of tracker may be employed at any place in the world as well as guarantees maximum efficiency in exerting solar energy. Concentrated solar power (CSP) processes using dual axis tracking include solar power structures and dish systems. Dual-axis tracking is incredibly significant in solar power tower processes because the angle error is important for longer distances between the mirrors in addition to the central receiver situated in the tower structure. Design for dual-axis tracking system is complex compared to single-axis tracking and operation is demanding [10]. Nevertheless, for large scale solar energy production dualaxis tracking is more fiscal.
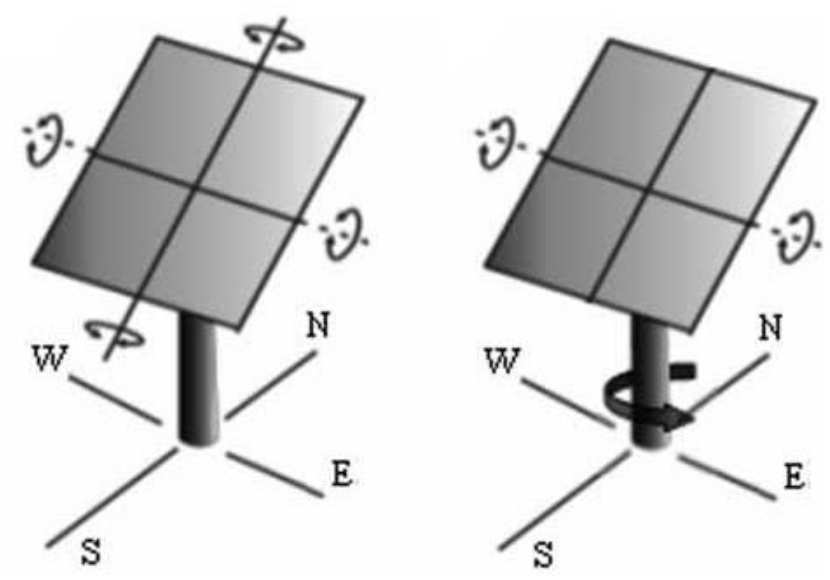

Figure 5: Dual Axis Solar Tracker

\subsection{Methods of Solar Tracking}

There are three methods of solar tracking.

- Active Tracking

- Passive Tracking

- Chronological Tracking

\subsubsection{Active Tracking}

The position of the sun is continuously determined by the sensors during the day. The sensor triggers the motion of motor or actuator in such a way so that the solar panel will always face the sun throughout the day. Active tracking is accurate with the help of sensors. But the main problem occurs when the sensors fail to discriminate between the measurements and give false trigger or miss original trigger during cloudy days.

\subsubsection{Passive Tracking}

Passive tracking method does not use sensors like active tracking. Instead of using sensors, a passive tracker moves in response to imbalance in pressure between two points at the ends of the tracker. This pressure imbalance is caused by heat from sun that creates gas pressure from compressed pressure moves the structure. This method does not have to rely on electrical sensors and requires negligible amount of power to operate. However, the mechanical design has to be very crucial to maintain accuracy.

\subsubsection{Chronological Tracking}

A chronological tracker is a timer-based tracking system. The structure is moved at a fixed rate throughout the day since the sun moves across the sky at a fixed rate of about 15 degree per hour. This method is better suitable for single axis tracking without sensors. For dual axis tracking a modified version can be implemented. The position of sun throughout the day can be calculated and set by the program implemented on the controller module. The solar tracker rotates according to data sent from control unit's memory of pre-stored data or calculated from given formula. This method of sun-tracking is accurate and reliable [11]. However, data storage, calculation continuous data transmission is power consuming and unnecessary rotation when sun light is too low can never be avoided. All the three methods are applicable with single axis and dual axis tracking system. Which method is best suitable, is determined by the location of installation, purpose of generation and demand of solar power. Modern trackers combines both the sensor controlled method and sensor less control method at the same time to increase efficiency.

\section{BASIC COMPONENTS OF SOLAR TRACKING SYSTEM}

A Solar tracker has several basic components. The major components are described here- 


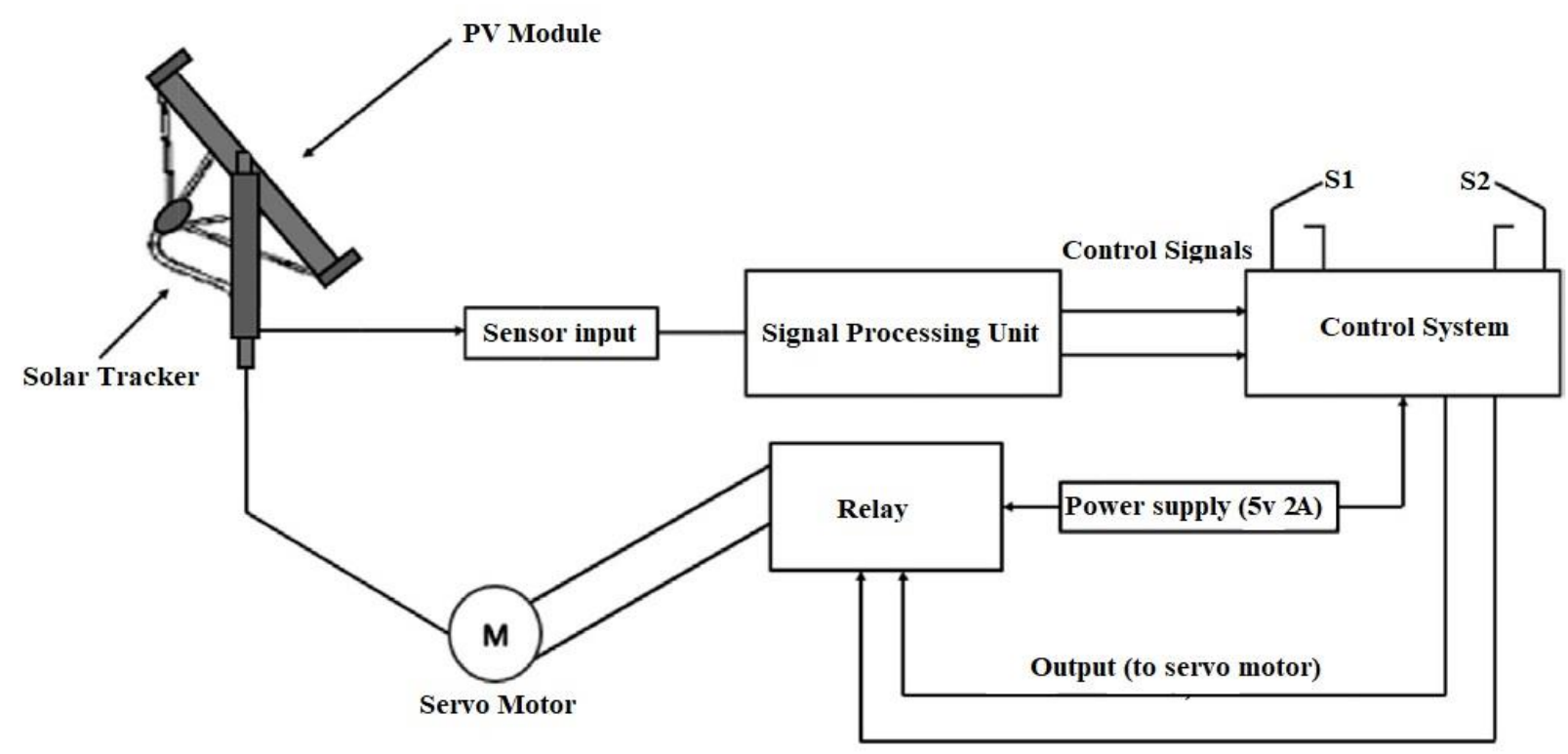

Figure 6:Solar Tracker Layout

2.1 Sun Tracking Algorithm: Solar tracking can have openloop control algorithm or closed-loop control algorithm. Open-loop control algorithm involves calculation of azimuth and altitude angle of sun on a purely mathematical platform based on astronomical references. The open-loop component is needed because the sun can be obscured by clouds, eliminating or distorting the feedback signals. Closed-loop control algorithm includes detection of the position of sun by real-time light-sensing method and is needed to eliminate errors due to variability in installation, assembly, calibration, and encoder mounting. These two methods can be combined together to keep balance between economic design and increased efficiency [12].

2.2 Tracker Control Unit: The control unit executes the sun tracking algorithm and necessary calculations. It can also coordinate the movement of the positioning system. A microprocessor or a computer can be used as the centre of control unit. It normally has command input and data output mechanism for interfacing. For the trackers placed in remote region automatic tracking control mechanism is best suitable.

2.3 Positioning System and Drive Mechanism: Positioning system moves the solar tracker according to the preference of control unit. It can be either electronic or hydraulic. Electrical systems utilize encoders and variable frequency drives or linear actuators to monitor the current position of the panel and move to desired positions. The drive mechanism includes mechanical devices- rotary motors, linear actuators, linear drives, hydraulic cylinders, swivel drives, worm gears, planetary gears, and threaded spindles. These drives can be of different types according to design method [13].

2.4 Sensors: Sensors are used to detect position of sun accurately. For open-loop system light sensors are implemented to correct calculation and mechanical errors. Closed-loop system solely depends on several light sensing devices. Light intensity detecting device includes photo- resistor, photo-transistor, solar cell etc. Solar power based stations also use temperature, pressure, humidity, wind velocity, solar irradiance and other necessary parameters monitoring systems with trackers.

\section{FACTORS LIMITING THE EFFICIENCY AND UTILIZATION OF SOLAR TRACKERS}

Generally, most of researchers have agreed that the solar trackers increase the overall efficiency of a PV panels and CSP systems. However, clear indications should be provided indicating where, when and how maximum power efficiency can be achieved. Consistent with the aforesaid, many problems have been identified and each of these problems has been addressed individually including miss-tracking and failure of the control systems and/or the electronics of the trackers among others. More importantly, the studies confirmed that most of the problems limiting the overall efficiency of both the fixed and tracking PV systems are similar. However, few problems are uniquely related to solar tracking systems such as those cited above. Moreover, different types of solar trackers present different advantages and challenges when considering their performance and efficiency, and studies have shown that tracking the sun position significantly increases the efficiency especially in cloudy days. For this reason, using ST could be the ideal way to boost up the efficiency and performance of both the PV panels and the CSP systems irrespective of what the weather looks like.

Similarly, it also has been shown that the number of axes used to track the sun have a significant impact of the overall efficiency of the solar radiation harvested by the module. It is studied the effect of tracking on the performance of PV modules based on the number of tracking axes for the system. They reaffirmed that the two axes tracking systems got higher ability to maintain their position without being affected by clouds or any other environmental conditions. Their double 
axis tracker produced $13.25 \%$ higher average output power than that of a fixed system [14]. An earlier study analyzed the difference in efficiencies between single axis and double axis ST and concluded that the double axis trackers are more efficient. They further added that the gain difference between these two types of trackers is often $3 \%$. All the above studies were performed in different areas with different climate conditions. The common point is that the overall efficiency of a solar tracker depends on the number of axes used but further added that both the climate conditions and the technology used could alter significantly the efficiencies of these systems especially in hot weather regions.

- Ambient and climatic conditions of the area

- Materials and technology

- Auxiliary equipment of the system

- Grid connection to the PV generator

- Costs and availability of solar tracking system

\subsection{ADVANTAGES OF SOLAR TRACKER}

The main advantage of using solar tracker is that it increases the availability of solar power from a location. A feasibility study was performed through an experiment prior to the design of solar tracker and it was found that power generation increased distinctively [15]. A 10 Watt solar panel will be used for the experiment. Open circuit voltage, close circuit current, voltage at different load were measured and maximum power points were determined throughout the daytime of a regular sunny day and the values will be plotted for comparison.

\section{RESULT}

Solar trackers are instruments which will be used to increase the amount of solar energy collected via PV systems via constant tracking of the position of the sun across the sky throughout the day. These methods are aesthetically attractive, environmentally friendly and are progressively becoming cost-efficient with the growth of the computer systems as well as control systems technologies. The integrated features bring these techniques appropriate as green and enormous scale domestic and industrial power generation processes [16]. In this article, different types of STS are summarized on their designs, thermal and electrical performances as well as the factors influencing the heat loss during operation.

Based on ideas of energy collection and the sun tracking techniques, ST can be divided into two categories viz. active types and passive types. These two major types of STS can, in turn, be divided into many other different categories based on a number of parameters such as the number of axes, the tracking directions and methods among others. Inactive STS, single/double-tracking modes are used. Each of these systems presents their own advantages; the double-axis STS provide better power stratifications while single axis processes are cheaper and less complex. the comparison between the energy returns of both tracking methods (single and double) with the fixed traditional PV systems open that the sun tracking system's energy return is always higher than that of the traditional fixed PV panels [17]. Although the double-axis tracking systems give better energy return when compared to other types of systems; the literature indicates that they suffer from high energy losses during their operation due to auxiliary units and moving joints. Serious problems occur when any of the sensors is shaded up since this phenomenon can cause an asymmetrical control signal leading to the malfunction of the entire system. Solar trackers are instruments which will be used to increase the amount of solar energy collected via PV systems via constant tracking of the position of the sun across the sky throughout the day. These methods are aesthetically attractive, environmentally friendly and are progressively becoming cost-efficient with the growth of the computer systems as well as control systems technologies. The integrated features bring these techniques appropriate as green and enormous scale domestic and industrial power generation processes. In this article, different types of STS are summarized on their designs, thermal and electrical performances as well as the factors influencing the heat loss during operation.

Based on ideas of energy collection and the sun tracking techniques, ST can be divided into two categories viz. active types and passive types. These two major types of STS can, in turn, be divided into many other different categories based on a number of parameters such as the number of axes, the tracking directions and methods among others. Inactive STS, single/double-tracking modes are used. Each of these systems presents their own advantages; the double-axis STS provide better power stratifications while single axis processes are cheaper and less complex [18]. the comparison between the energy returns of both tracking methods (single and double) with the fixed traditional PV systems open that the sun tracking system's energy return is always higher than that of the traditional fixed PV panels. Although the double-axis tracking systems give better energy return when compared to other types of systems; the literature indicates that they suffer from high energy losses during their operation due to auxiliary units and moving joints. Serious problems occur when any of the sensors is shaded up since this phenomenon can cause an asymmetrical control signal leading to the malfunction of the entire system.

\section{CONCLUSIONS}

It is evident from the above study the use of solar trackers will certainly increase the efficiency of SPV systems but proper care is required while installing solar trackers with these systems. Problems such as failure of solar tracker also need equal attention while installing them. Whether to install active or passive solar trackers is also a question which needs to be explored further. This study also tried to analyze all the important factors which are required for the optimization of solar trackers.

\section{REFERENCES}

[1] N. Gupta, N. Patel, R. Ladha, and D. P. Saini, "A novel autoscaling variable perturbation size maximum power point tracker applied to photovoltaic (PV) system," Int. Trans. Electr. Energy Syst., no. June 2019, pp. 1-17, 2020. 
[2] P. A. C. Rocha, S. P. Mendonça, T. T. G. Watanabe, M. E. V. da Silva, and R. J. P. Lima, "Analysis of different tracking intervals for Parabolic Trough Collectors for water disinfestation in agricultural applications," Semin. Ciências Agrárias, vol. 41, no. 1, p. 7, 2020.

[3] K. Aygül, M. Cikan, T. Demirdelen, and M. Tumay, "Butterfly optimization algorithm based maximum power point tracking of photovoltaic systems under partial shading condition," Energy Sources, Part A Recover. Util. Environ. Eff., vol. 00, no. 00, pp. 1-19, 2019.

[4] N. Kumar, B. Singh, and B. K. Panigrahi, "LLMLF-Based Control Approach and LPO MPPT Technique for Improving Performance of a Multifunctional Three-Phase Two-Stage Grid Integrated PV System," IEEE Trans. Sustain. Energy, vol. 11, no. 1, pp. 371-380, 2020.

[5] A. A. A. Radwan and Y. A. R. I. Mohamed, "Grid-Connected WindSolar Cogeneration Using Back-to-Back Voltage-Source Converters,' IEEE Trans. Sustain. Energy, vol. 11, no. 1, pp. 315-325, 2020

[6] A. K. Mishra and B. Singh, "Self-governing single-stage photovoltaic water pumping system with voltage balancing control for a four-phase SRM drive," IET Electr. Power Appl., vol. 14, no. 1, pp. 119-130, 2020.

[7] L. M. Fernández-Ahumada, J. Ramírez-Faz, R. López-Luque, M. VaroMartínez, I. M. Moreno-García, and F. Casares de la Torre, "A novel backtracking approach for two-axis solar PV tracking plants," Renew. Energy, vol. 145, pp. 1214-1221, 2020.

[8] S. Ahmadnia, E. Tafehi, and F. Shakhsi Dastgahian, "Optimal Placement and Sizing for Solar Farm with Economic Evaluation, Power Line Loss and Energy Consumption Reduction," IETE J. Res., vol. 0, no. 0, pp. 1-16, 2019.

[9] A. Chaysaz, S. R. M. Seyedi, and A. Motevali, "Effects of different greenhouse coverings on energy parameters of a photovoltaic-thermal solar system," Sol. Energy, vol. 194, no. November, pp. 519-529, 2019.

[10] M. Dhimish, “70\% Decrease of Hot-Spotted Photovoltaic Modules Output Power Loss Using Novel MPPT Algorithm," IEEE Trans. Circuits Syst. II Express Briefs, vol. 66, no. 12, pp. 2027-2031, 2019.

[11] A. K. Singh, K. A. Chinmaya, and M. Badoni, "Solar PV and grid based isolated converter for plug-in electric vehicles," IET Power Electron., vol. 12, no. 14, pp. 1-9, 2019.

[12] R. Sharma and A. Das, "Enhanced Active Power Balancing Capability of Grid-Connected Solar PV Fed Cascaded H-Bridge Converter," IEEE J. Emerg. Sel. Top. Power Electron., vol. 7, no. 4, pp. 2281-2291, 2019.

[13] X. Qian et al., "Artificial phototropism for omnidirectional tracking and harvesting of light," Nat. Nanotechnol., vol. 14, no. 11, pp. 1048$1055,2019$.

[14] D. T. Cotfas and P. A. Cotfas, "Multiconcept Methods to Enhance Photovoltaic System Efficiency," Int. J. Photoenergy, vol. 2019, 2019.

[15] T. Zheng, F. Zheng, X. Rui, X. Ji, and K. Niu, "A novel ultralight dish system based on a three-extensible-rod solar tracker," Sol. Energy, vol. 193, no. June, pp. 335-359, 2019

[16] S. Chauhan and B. Singh, "Grid-interfaced solar PV powered electric vehicle battery system with novel adaptive digital control algorithm," IET Power Electron., vol. 12, no. 13, pp. 3470-3478, 2019.

[17] T. Lee, J. Kim, S. Cho, S. Pyo, K. Song, and J. Lee, "Planar-type concentrating photovoltaics with cylindrical lenses directly integrated with thin flexible GaAs solar cells," Prog. Photovoltaics Res. Appl., vol. 28, no. 1, pp. 71-78, 2020.

[18] M. Premkumar and R. Sowmya, "An effective maximum power point tracker for partially shaded solar photovoltaic systems," Energy Reports, vol. 5, pp. 1445-1462, 2019. 